\title{
Europäische Integration und nationaler Parteien- wettbewerb: theoretische Überlegungen und empirische Befunde am Beispiel der Eurokrise
}

von Oskar Niedermayer

Der Beitrag weist zunächst auf der theoretischen Ebene mögliche Formen einer Einbindung der europäischen Integration in die Konfliktstruktur nationaler Parteienwettbewerbe aus und sucht im Anschluss die konkreten Ausformungen einer solchen Einbindung im Rahmen von Parlamentswahlkämpfen in ausgewählten Ländern der Eurozone aufzuzeigen. Die Ergebnisse dokumentieren, dass seit Beginn der Eurokrise unterschiedliche Auswirkungen auf den nationalen Parteienwettbewerb zu beobachten sind. So wurde in zwei Ländern eine neue, eigenständige Konfliktlinie in die parteipolitische Auseinandersetzung integriert. In einem weiteren Land spielte die europäische Entwicklung im Wahlkampf keine große Rolle, während in der Mehrheit der untersuchten Fälle die Parteien in ihrem framing der Eurokrise primär deren sozio-ökonomische Auswirkungen akzentuierten, sodass eine Integration in den bestehenden sozio-ökonomischen Sozialstaatskonflikt erfolgte. Einige Parteien, vor allem am rechten Rand des politischen Spektrums, thematisierten allerdings durchaus auch sozio-kulturelle Aspekte der Krise.

In a first step, this contribution sheds light on the theoretically conceivable ways of including the factor "European integration" into the conflict structures of multi-party competition at the national level. Subsequently, the empirically identifiable patterns of including the Euro crisis in the election campaigns of selected Eurozone countries are outlined. The results indicate that the crisis has influenced the party-political arenas in different ways. In two countries, a new and independent dimension of conflict has been established, while in one further country, the crisis played no important role at all. However, in the majority of cases, existing parties framed the crisis primarily by accentuating the socio-economic dimension, thereby integrating it into pre-existing conflict structures. Some right-wing parties stressed socio-cultural aspects instead.

\section{Einleitung}

In Deutschland spielte die europäische Integration vor der spätestens 2010 erkennbaren Eurokrise im nationalen Parteienwettbewerb keine große Rolle. Auch für die Bundestagswahl 2009 galt weiterhin der Befund, dass die Einbindung der Bundesrepublik in die EU, ,nicht zu gravierenden Veränderungen der einzelnen 
Charakteristika des Parteiensystems geführt"1 hat. Das Europa-Thema blieb für die Wählerinnen und Wähler von geringer Relevanz, unter den Bundestagsparteien gab es ,in zentralen Fragen der Europapolitik ... einen breiten Konsens“, der grundsätzlich, ,wenngleich mit erheblichen Abstrichen“2, auch die Linkspartei mit einschloss, dezidiert pro- oder anti-europäische Parteigründungen ${ }^{3}$ bzw. europafeindliche rechtspopulistische oder rechtsextreme Parteien konnten keine Wahlerfolge erzielen und selbst die Europawahlen wurden einschließlich der Wahl von 2009 ,durch die nationale Politikarena dominiert“44.

Inzwischen hat die Eurokrise in Deutschland jedoch inzwischen zu einer Politisierung des Themas europäische Integration geführt und das über Jahrzehnte geltende zwischenparteiliche Konsensprinzip aufgeweicht. ${ }^{5}$ Hinzu kommt, dass im Februar 2013 mit der „Alternative für Deutschland“ (AfD) eine Partei gegründet wurde, die sich als klare Alternative zur Euro-Politik der anderen Parteien versteht. Sie forderte in ihrem Wahlprogramm eine ,geordnete Auflösung des Euro-Währungsgebietes“ und die „Wiedereinführung nationaler Währungen oder die Schaffung kleinerer und stabilerer Währungsverbünde." Auch die Wiedereinführung der DM dürfe kein Tabu sein. Durch eine Änderung der Europäischen Verträge soll jedem Staat ein Ausscheiden aus dem Euro ermöglicht werden, und Deutschland solle dieses Austrittsrecht aus dem Euro erzwingen, indem es weitere Hilfskredite des ESM mit seinem Veto blockiert. Europapolitisches Ziel der AfD ist „ein Europa souveräner Staaten mit einem gemeinsamen Binnenmarkt“, einer Zurückverlagerung von Gesetzgebungskompetenzen auf die Mitgliedstaaten und insbesondere ,dem uneingeschränkten Budgetrecht der nationalen Parlamente.“ Ein zentralisierter Europastaat wird entschieden abgelehnt. Zudem will man sich „für eine Reform der EU stark machen, um die Brüsseler Bürokratie abzubauen und Transparenz und Bürgernähe zu fördern." Nach einem anfängli-

1 Niedermayer, O.: Europäisierung des deutschen Parteiensystems?, in: Brettschneider, F./van Deth, J./Roller, E. (Hg.): Europäische Integration in der öffentlichen Meinung, Opladen, 2003, 253-277, hier 273.

2 Pehle, H./Sturm, R.: Die europäische Integration - ein relevanter Bezugsrahmen des nationalen Parteienwettbewerbs?, in: Zeitschrift für Politik, 57/3 (2010), 294-306, hier 299.

3 Die pro-europäischen Partei „Europapartei (Europäische Föderalistische Partei)“ erhielt 1969 nur 0,2 Prozent, die integrationskritischen Parteien „Bund Freier Bürger - Offensive für Deutschland“ bzw. „Initiative Pro D-Mark - neue liberale Partei“ erhielten 1998 0,2 bzw. 0,9 Prozent.

4 Niedermayer, O.: Die Wahl zum Europäischen Parlament vom 7. Juni 2009 in Deutschland: SPDDebakel im Vorfeld der Bundestagswahl, in: Zeitschrift für Parlamentsfragen 40/4 (2009), 711-731, hier 720.

5 Vgl. Wimmel, A.: Deutsche Parteien in der Euro-Krise: Das Ende des Konsensprinzips?, in: Integration, 35/1 (2012), 19-34. 
chen Medienhype, der bis Mitte April 2013 anhielt, wurde es deutlich ruhiger um die Partei, konnte sie in den Umfragen nicht mehr als Zustimmungswerte in Höhe von 2-3 Prozent erzielen. Dies war vor allem dem Umstand geschuldet, dass sie - obwohl sie sich selbst nicht als Ein-Themen-Partei sieht und sich zu allen die öffentliche Diskussion prägenden Fragen äußert ${ }^{6}$ - in der Öffentlichkeit als Anti-Euro-Partei wahrgenommen wurde und die Eurokrise lange Zeit im Wahlkampf keine Rolle spielte. Nachdem sich Bundesfinanzminister Wolfgang Schäuble Mitte August 2013 zu einem möglichen neuen Rettungspaket für Griechenland äußerte und die Oppositionsparteien daraufhin mit dem Vorwurf reagierten, die Regierung verschleiere gegenüber den Bürgern die wahren Kosten der Eurorettung, erreichte die AfD Anfang September in einer Umfrage 4 Prozent und erhielt wieder etwas mehr Aufmerksamkeit, verpasste letztlich aber knapp den Einzug in den Bundestag.

In vielen anderen Ländern der EU stellte sich die Situation schon seit dem Maastrichter Vertrag von 1991 anders dar: Fragen der europäischen Integration beeinflussten das Wahlverhalten bei nationalen Wahlen ${ }^{7}$ und waren relevante Themen im nationalen Parteienwettbewerb. ${ }^{8}$ Mit der Eurokrise potenzierte sich dies und führte zu einer Reihe von Regierungswechseln und vorgezogenen Parlamentsneuwahlen. Es bietet sich daher an, sich theoretisch wie empirisch der Frage nach dem Verhältnis von europäischer Integration und nationalem Parteienwettbewerb zuzuwenden. Der folgende Beitrag analysiert zunächst auf der theoretischen Ebene die Einbindung der europäischen Integration in die Konfliktstruktur der nationalen Parteienwettbewerbe und untersucht im Anschluss die konkrete Ausformung dieser Einbindung in die Parlamentswahlkämpfe ausgewählter Länder der Eurozone.

\section{Die Einbindung der europäischen Integration in die Konflikt- struktur des nationalen Parteienwettbewerbs}

In der Parteienforschung wird seit Jahrzehnten über die Anzahl und Art der grundlegenden Konfliktlinien diskutiert, die den nationalen Parteienwettbewerb

6 Die generelle politische Einordnung der Partei durch politische Beobachter ist umstritten. Sie reicht von konservativ bis hin zu rechtspopulistisch.

7 Vgl. z. B. Gabel, M.: European Integration, Voters and National Politics, in: West European Politics 23/4 (2000), S. 52-72 und de Vries, C. E.: EU Issue Voting: Asset or Liability?, in: European Union Politics 11/1 (2010), 89-117.

8 Vgl. etwa Benoit, K./Laver, M.: Party Policy in Modern Democracies, New York, 2006. 
prägen. Ausgangspunkte der Auseinandersetzungen sind zum einen das räumliche Modell des Parteienwettbewerbs im Rahmen der ökonomischen Theorie der Demokratie von Anthony Downs und zum anderen die Theorie gesellschaftlicher Konfliktlinien (cleavages) von Seymour M. Lipset und Stein Rokkan. ${ }^{9}$ Downs sah den Parteienwettbewerb durch eine einzige Konfliktlinie geprägt: den LinksRechts-Konflikt, konkretisiert anhand des Wertekonflikts über die Frage der Staatsintervention in die Ökonomie. Lipset und Rokkan dagegen machten vier gesellschaftliche Konfliktlinien aus, durch deren Politisierung sich die Parteiensysteme Westeuropas Mitte des 19. Jahrhunderts herausbildeten: den Klassenkonflikt, den Stadt-Land-Konflikt, den Kirche-Staat-Konflikt und den ZentrumPeripherie-Konflikt. Eine Analyse der in den darauf folgenden Jahren kontrovers geführten Diskussion ergibt folgendes Tableau von parteipolitischen Konfliktlinien, die in unterschiedlicher Intensität und Zusammensetzung den Parteienwettbewerb geprägt haben bzw. prägen können: ${ }^{10}$

- Sozio-ökonomischer Bereich (Konflikte um die Rolle des Staates in der Ökonomie): Sozialstaatskonflikt (Konflikt um die Rolle des Staates bei der Verteilung von Gütern und Dienstleistungen: soziale Gerechtigkeit vs. Marktfreiheit), Eigentumskonflikt (Konflikt um die Rolle des Staates bei der Produktion von Gütern und Dienstleistungen: Staatseigentum vs. Privateigentum an Produktionsmitteln), Stadt-Land-Konflikt (Konflikt zwischen sekundärem/tertiärem und primärem Sektor).

- Sozio-kultureller Bereich (Konflikte um die Gestaltung des menschlichen Zusammenlebens): Kirche-Staat-Konflikt (Konflikt zwischen katholischer Kirche und Staat), Religionskonflikt (Konflikt zwischen religiösen und säkularen Wertorientierungen), Libertarismus-Autoritarismus-Konflikt (Konflikt zwischen libertären und autoritären Wertorientierungen).

- Politisch-konstitutioneller Bereich (Konflikte um die Gestaltung der politischen Ordnung): Systemkonflikt (Konflikt zwischen demokratischen und undemokratischen Wertorientierungen).

Hinzu kommen der Ökonomie-Ökologie-Konflikt (um die Ausrichtung der Politik an ökonomischen oder ökologischen Notwendigkeiten), der sowohl sozioökonomische als auch sozio-kulturelle Implikationen haben kann, sowie der im sozio-kulturellen Bereich wurzelnde, aber auf die beiden anderen Bereiche aus-

9 Vgl. Downs, A.: An Economic Theory of Democracy, New York, 1957 und Lipset, S. M./Rokkan, S: Cleavage Structures, Party Systems, and Voter Alignments: An Introduction, in: dies. (Hg.): Party Systems and Voter Alignments. Cross-national Perspectives, New York, 1967, 1-64.

10 Vgl. Niedermayer, O.: Gesellschaftliche und parteipolitische Konfliktlinien, in: Kühnel, S./Niedermayer, O./Westle, B. (Hg.): Wähler in Deutschland. Sozialer und politischer Wandel, Gender und Wahlverhalten, Wiesbaden, 2009, 30-67. 
strahlende Zentrum-Peripherie-Konflikt (zwischen der Mehrheit und regionalen ethnischen, sprachlichen, konfessionellen oder sonstigen Minderheiten). Viele empirische Analysen haben gezeigt, dass in Deutschland und vielen anderen europäischen Ländern aus diesem Tableau vor allen zwei parteipolitische Konfliktlinien relevant sind; im sozio-ökonomischen Bereich der Sozialstaatskonflikt und im sozio-kulturellen Bereich der Libertarismus-Autoritarismus-Konflikt.

Vor diesem Hintergrund lässt sich die europäische Integration auf sehr unterschiedliche Weise in die Konfliktstruktur des nationalen Parteienwettbewerbs einbinden. Zunächst kann man in der Tradition von Downs unterschiedliche Positionen zur europäischen Integration als spezifische Ausformung des LinksRechts-Konflikts sehen, wo die Befürworter einer sozialstaatlichen Marktregulierung den Befürwortern der Marktfreiheit (auf der europäischen Ebene) gegenüberstehen. ${ }^{11}$ Sieht man den Parteienwettbewerb in der Tradition von Lipset/Rokkan als durch eine mehrdimensionale Konfliktstruktur bestimmt, wie hier angenommen, so bieten sich grundsätzlich zwei Möglichkeiten an:

Zum einen kann der europäische Integrationskonflikt als eine neue, eigenständige Konfliktlinie in der parteipolitischen Konfliktstruktur gesehen werden: Man kann ihn als bereichsübergreifenden Zentrum-Peripherie-Konflikt neuer Art konzeptualisieren, in dem die Befürworter einer supranationalen Integration von Nationalstaaten den Befürwortern nationaler Autonomie gegenüberstehen, ${ }^{12}$ man kann ihn auf den sozio-kulturellen Bereich begrenzt als LibertarismusAutoritarismus-Konflikt um die Frage der nationalen Identität ansehen, in dem sich ,,green/alternative/libertarian (or gal) “ und ,traditionalism/authority/nationalism (or tan) " Parteien gegenüberstehen, ${ }^{13}$ oder man kann ihn schließlich als Manifestation eines generellen Globalisierungskonflikts mit sozio-ökonomischen und sozio-kulturellen Dimensionen konzeptualisieren, in dem sich die Gewinner und die Verlierer der Globalisierung gegenüberstehen. ${ }^{14}$ Zum anderen können die unterschiedlichen policy-Positionen der Parteien zu den mit der euro-

11 Vgl. Hooghe, L./Marks, G.: The Making of a Polity: The Struggle over European Integration, in: Kitschelt, H./Lange, P./Marks, G./Stephens, J. (Hg.): Continuity and Change in Contemporary Capitalism, Cambridge, 1999, 70-97.

12 Vgl. Niedermayer, O.: Europäisierung, a.a.O., 259.

13 Vgl. Hooghe, L./Marks, G.: A Postfunctionalist Theory of European Integration: From Permissive Consensus to Constraining Dissensus, in: British Journal of Political Science 39/1 (2008), 1-23, hier 16.

14 Vgl. Kriesi, H.: Rejoinder to Liesbet Hooghe and Gary Marks, 'A Postfunctional Theory of European Integration: From Permissive Consensus to Constraining Dissensus', in: British Journal of Political Science, 40/1 (2009), 221-224, und Kriesi, H./Grande, E./Lachat, R./Dolezal, M./Bornschier, S./Frey, T. (Hg.): West European Politics in the Age of Globalisation, Cambridge, 2008. 
päischen Integration verbundenen Fragen, ${ }^{15}$ etwa der Eurorettung, in die bestehende parteipolitische Konfliktstruktur der einzelne Staaten integriert werden, ${ }^{16}$ indem man sie als thematische Konkretisierung schon existierender Konfliktlinien begreift.

$\mathrm{Zu}$ betonen ist, dass nicht jeder zwischenparteiliche Konflikt als parteipolitischer cleavage anzusehen ist. Um als solcher zu gelten, muss sich der Konflikt zum einen auf diametral entgegengesetzte Zielvorstellungen und nicht nur auf Differenzen in den Mitteln und Wegen zur Erreichung eines von allen geteilten Ziels beziehen, zum anderen muss er parteipolitisch in relevanter Weise repräsentiert sein. Wenn man - wie es hier geschehen soll - die europäische Integrationsfrage als bereichsübergreifenden Zentrum-Peripherie-Konflikt neuer Art (um die Frage nationaler Autonomie vs. supranationaler Integration) konzeptualisiert, dann lässt sich die Entscheidung, ob die europäische Integration in einem EUMitgliedsland eine relevante eigenständige Konfliktlinie im nationalen Parteienwettbewerb darstellt, anhand von zwei Kriterien treffen: Zum einen muss der Konflikt durch Parteien repräsentiert sein, die sich nicht nur um die Art und Weise streiten, wie die europäische Integration ausgestaltet sein soll, sondern durch solche, die für oder gegen die Einbindung ihres Landes in einen europäischen Verbund sind. Insofern muss es vor allem auch eine den Austritt des Landes aus der EU propagierende Partei geben, die explizit als Anti-EU-Partei gegründet wurde oder zumindest dieses Thema im untersuchten nationalen Wahlkampf in den Mittelpunkt stellt. Zum anderen müssen beide Seiten des Konflikts durch relevante Parteien im Parteiensystem repräsentiert sein, was durch ihre parlamentarische Repräsentation operationalisiert werden kann.

Sind diese Bedingungen nicht erfüllt, kann es natürlich aufgrund unterschiedlicher policy-Positionen der Parteien zu parteipolitischen Konflikten in diesem Bereich kommen. Die Konflikte sind in diesem Fall jedoch in die bestehende parteipolitische Konfliktstruktur des Landes integriert, d.h. sie stellen Konkretisierungen schon bestehender cleavages dar. Um die Frage zu beantworten, wie diese Integration konkret aussieht, muss zunächst die parteipolitische Konfliktstruktur bestimmt werden; in einem zweiten Schritt ist dann zu analysieren, welcher/welchen Konfliktlinie(n) das jeweils relevante Sachthema aus dem Bereich

15 Empirische Analysen unterscheiden 20 unterschiedliche Themenbereiche im EU policy space, vgl. Pennings, P.: The Dimensionality of the EU Policy Space, in: European Union Politics 3/1 (2002), 5980.

16 Vgl. z.B. Sitter, N.: Opposing Europe: Euro-Scepticism, Opposition and Party Competition, SEI Working Paper, Brighton 2002. 
der europäischen Integration im nationalen Parteienwettbewerb zugeordnet wird. Letzteres kann etwa dadurch geschehen, dass das framing ${ }^{17}$ des Themas durch die Parteien in den Wahlkämpfen zu nationalen Parlamentswahlen untersucht wird.

Wir gehen aufgrund der bisherigen empirischen Evidenz davon aus, dass die Konfliktstruktur der EU-Staaten mindestens zweidimensional ist und durch den sozio-ökonomischen Sozialstaats- und den sozio-kulturellen Libertarismus-Autoritarismus-Konflikt geprägt wird. Auf welchen oder welche dieser parteipolitischen cleavages sich die Argumente der Parteien zur Unterstützung oder Kritik der europäischen Integration beziehen, wie also das framing aussieht, hängt von der jeweiligen Parteienfamilie ab, wie die folgende Zusammenfassung der bisherigen Forschung verdeutlicht:

While the left tends to embrace the cultural dimension and is more critical of economic aspects of integration, the right supports market liberalisation but has different opinions on cultural issues. ... Parties of the centre-left are typically in favour of diminishing cultural boundaries, and thus we expect them to use multicultural universalist arguments to support European integration. They are, however, in favour of more (economic) integration if particular projects focus on the (re-)regulation of the common market. ... The communists and left socialists oppose European integration energetically, deeming it a neo-liberal project that endangers the achievements of the welfare state. ... In the rare instances where they demonstrate support of European integration, they stress the potential to lower cultural boundaries, invoking multicultural-universalist frames. Green parties ... also support political and cultural integration strongly, and thus are likely to employ multicultural-universalist justifications. On the other side of the political spectrum, liberal parties often support further integration as long as it is in the service of market liberalisation - opposing further integration when political or cultural aspects are concerned. ... Christian democratic and conservative parties in particular can be differentiated from other right-wing parties insofar as they ... clearly support political and cultural integration. ... At the far right, European integration is rejected in all its aspects. For these parties, political and cultural integration constitutes a threat to national traditions and to sovereignty. ${ }^{18}$

Zudem tendieren nationale Regierungsparteien in Fragen der europäischen Integration meist zu einer moderateren Haltung als Oppositionsparteien, insbeson-

17 Framing ist definiert als "to select some aspects of a perceived reality and make them more salient in a communicating text, in such a way as to promote a particular problem definition, causal interpretation, moral evaluation, and/or treatment recommendation" (Entman, R.M.: Framing: Toward Clarification of a fractured Paradigm, in: Journal of Communication 43/4 (1993), 51-58, hier 52).

18 Helbling, M./Hoeglinger, D./Wüest, B.: How political parties frame European integration, in: European Journal of Political Research 49/4 (2010), 496-521, hier $502 \mathrm{f}$. 
dere denjenigen an den Rändern des ideologischen Spektrums.

In der folgenden framing-Analyse ausgewählter Wahlkämpfe wird daher der Frage nachgegangen, ob die Eurokrise zur Etablierung einer zusätzlichen, eigenständigen Konfliktlinie im Sinne eines neuen Zentrum-Peripherie-Konflikts um die Frage nationaler Autonomie vs. supranationaler Integration geführt hat, oder ob die Parteien der unterschiedlichen Parteienfamilien im Rahmen ihrer Wahlkampfkommunikation ihre Position zur Eurokrise und den Maßnahmen zu ihrer Bewältigung in ihre Konfliktposition (im Sozialstaats- und/oder LibertarismusAutoritarismus-Konflikt) integriert haben.

\section{Das framing der Eurokrise in den Wahlkämpfen zu nationalen Parlamentswahlen}

Die schon seit Ende 2009 schwelende Eurokrise, besser als Finanzwirtschaftsund Staatsschuldenkrise im Euroraum bezeichnet, wurde spätestens im April 2010 öffentlich sichtbar, als Griechenland zur Abwendung eines Staatsbankrotts bei der Europäischen Union und dem Internationalen Währungsfonds um Hilfe bat. Von diesem Zeitpunkt bis zum März 2013 fanden in 14 der 17 die Eurozone umfassenden Staaten insgesamt 17 Wahlen zum jeweiligen nationalen Parlament statt. ${ }^{19}$ Eine Analyse all dieser Wahlen ist hier nicht möglich. Es wurde daher eine Auswahl getroffen, die - im Sinne des most-different-systems-approach systematisch die unterschiedliche Situation der Länder (vor allem Geber- vs. Nehmerländer), die Diversität ihrer Parteiensysteme und den Krisenverlauf widerspiegelt. Behandelt werden die Parlamentswahlen 2011 in Irland, Finnland und Spanien, 2012 in der Slowakei, in Griechenland (zwei Wahlen) und den Niederlanden sowie die Wahl im Februar 2013 in Italien.

\section{Irland: die Unterhauswahl am 25. Februar 2011}

Irland erlebte über längere Zeit einen außergewöhnlich steilen Wirtschaftsaufschwung, der jedoch vor allem auf der Finanzindustrie basierte und daher von der internationalen Finanzkrise 2008 besonders hart getroffen wurde. Die staatlichen Einnahmen brachen zusammen, die Regierung aus Fianna Fáil (FF) und Green Party sprach im Oktober 2008 staatliche Stützungsgarantien für das zusammenbrechende Bankensystem aus und das Haushaltsdefizit stieg in der Fol-

19 In Griechenland, den Niederlanden und der Slowakei wurde jeweils zweimal gewählt. Keine Parlamentswahlen fanden im Untersuchungszeitraum in Deutschland, Luxemburg und Österreich statt. 
gezeit so dramatisch, dass der Staatsbankrott drohte. Im November 2010 bat die Regierung die EU um Hilfe und unterzeichnete schließlich ein Rettungspaket der ,Troika‘ aus Europäischer Kommission (EU), Europäischer Zentralbank (EZB) und Internationalem Währungsfonds (IWF) in Höhe von 85 Mrd. Euro, das dem Land einen strikten Sparkurs auferlegte. Anfang 2011 brach die Regierung auseinander und es kam zu einer vorgezogenen Unterhauswahl am 25. Februar 2011, die zum ersten durch die Finanzkrise im Euroraum bewirkten Regierungswechsel führte, da die Wählerschaft das Vertrauen in das Krisenmanagement der Regierung schon seit längerer Zeit verloren hatte.

Die Wahlkampagne war eindeutig von sozio-ökonomischen Themen dominiert. Neben dem Thema Arbeitslosigkeit wurde die Diskussion vor allem von der Frage beherrscht, wie mit dem Rettungspaket umzugehen sei. ${ }^{20}$ Die FF versuchte unter neuer Führung ihre Verantwortung für das bisherige Krisenmanagement vergessen zu machen, konnte aber als damaliger Verhandlungsführer das Verhandlungsergebnis mit der Troika nicht infrage stellen. Die Gegenposition vertrat die linke Sinn Féin (SF) mit ihrer Forderung der einseitigen Aufkündigung des Rettungspakets und Abwicklung der Banken ohne Gläubigerentschädigung. Der SF-Vorsitzende Gerry Adams verkündete sogar in einem Interview, dass er Verhandlungen über eine Regierungsteilnahme nur unter der Bedingung führen würde, dass Irland aus der EU austritt. ${ }^{21}$ Die SF ist von ihrer Geschichte her jedoch nicht als Anti-EU-Partei zu klassifizieren. Ihre Wurzeln liegen im irischen Unabhängigkeitskampf und sie war in den Achtzigerjahren der politische Arm der IRA. Zudem spielte die Forderung nach einem EU-Austritt in ihrer Kampagne keine zentrale Rolle. Die größte Oppositionspartei Fine Gael (FG), wie die FF eine marktliberale Partei, und die Sozialdemokraten (LP) kritisierten die Bedingungen des Pakets und kündigten im Falle einer Regierungsübernahme Nachverhandlungen mit dem Ziel an, den Kreditzinssatz herabzusetzen. Zur Erreichung der Sparziele setzte - im Einklang mit ihrer jeweiligen Positionierung im Sozialstaatskonflikt - die FG vor allem auf Ausgabenkürzungen, während die LP stärker auf Steuererhöhungen abstellte. Die Wahl veränderte das irische Parteiensystem dramatisch: Die FF, die seit ihrem ersten Wahlsieg 1932 im Unterhaus stets die größte Fraktion gestellt hatte und meist allein regieren konnte,

20 Vgl. z.B. Courtney, M./Gallagher, M.: The parliamentary election in Ireland, February 2011, in: Electoral Studies, 31/1 (2012), 231-234; Kirby, P.: Wahlanalyse Irland 2011, FES-Reihe Internationale Politikanalyse, Berlin, 2012.

21 Vgl. Deloy, C.: General Elections in Ireland, 25th February 2011, FRS-Reihe European Elections monitor, Brüssel, 2011, 5. 
musste sich diesmal nicht nur der FG geschlagen geben, sondern fiel hinter der LP sogar auf den dritten Platz zurück. Viertstärkste Partei blieb die SF. Die Frage, ob die SF ihre Austrittsforderung aus der EU in Regierungsverhandlungen tatsächlich vertreten hätte, stellte sich nicht, da FG und LP die Regierung bildeten.

\section{Finnland: die Parlamentswahl am 17. April 2011}

Im Gegensatz zur irischen war die finnische Wahl keine vorgezogene Neuwahl, obwohl die Regierung aus Zentrumspartei (KESK), Nationaler Sammlungspartei (KOK), dem Grünen Bündnis (VIHR) und der Schwedischen Volkspartei (RKP) von einer vor allem die KESK und die KOK betreffenden Parteienfinanzierungsaffäre erschüttert wurde. Die Affäre führte im Juni 2010 zum Rücktritt des Premierministers Matti Vanhanen (KESK), der durch seine Parteifreundin Mari Kiviniemi ersetzt wurde. ${ }^{22}$

Neben der Finanzierungsaffäre wurde die Frühphase des Wahlkampfes von innenpolitischen Konkretisierungen der beiden zentralen parteipolitischen Konfliktlinien, dem Sozialstaatskonflikt und dem Libertarismus-Autoritarismus-Konflikt, bestimmt. ${ }^{23}$ Im sozio-ökonomischen Bereich war es der Streit um eine Erhöhung der Mehrwertsteuer zur Reduktion der Staatsschulden, die von den Regierungsparteien befürwortet und von den Oppositionsparteien abgelehnt wurde, während im sozio-kulturellen Bereich die Haltung gegenüber sexuellen Minderheiten (konkretisiert anhand von gleichgeschlechtlichen Ehen) im Zentrum stand; hier verlief die die Front zwischen KESK, KOK, Christdemokraten (KD) und den Wahren Finnen (PS) auf der einen und den Sozialdemokraten (SDP), dem Linksbund (VAS), den Grünen (VIHR) und der RKP auf der anderen Seite. In der Schlussphase des Wahlkampfs wurde dann auch Finnlands Beteiligung an den Maßnahmen zur Sicherung des Euro und zur Stützung Griechenlands, Irlands und Portugals zum Thema. KESK, KOK, VIHR und RKP unterstützten die Europäische Finanzstabilisierungsfazilität (ESFS) und den Europäischen Stabilitätsmechanismus (ESM), der nach den Gipfelbeschlüssen von Ende März 2011 die ESFS ab 2013 ablösen sollte, während die PS, der VAS und die KD sich dagegen aussprachen und die gewöhnlich pro-europäische SDP die Rettungsfonds nur akzeptieren wollte, wenn die Banken mit einbezogen

22 Vgl. Deloy, C.: General Elections in Finland, 17th April 2011, FRS-Reihe European elections monitor, Brüssel, 2011.

23 Vgl. Nurmi, H./Nurmi, L.: The parliamentary election in Finland, April 2011, in: Electoral Studies 31/1 (2012), 234-238. 
würden. ${ }^{24}$ Vor allem für die wegen ihrer konservativ-autoritären Positionen im sozio-kulturellen Bereich meist als rechtspopulistisch eingestuften Wahren Finnen $^{25}$ war - neben ihrem Standardthema Integration - die Ablehnung der EUFinanzhilfen in der Schlussphase das Hauptmobilisierungsthema. Die Botschaft ihres Vorsitzenden Timo Soini, der sich erfolgreich als Mann des Volkes und Verteidiger der Armen gegen das Establishment darstellte, war schlicht: Die finnischen Steuerzahler sollen nicht für die Fehler anderer Länder bezahlen müssen. Mit dieser Strategie gelang der Partei ein Erdrutschsieg: Sie konnte ihren Mandatsanteil von 2,5 auf 19,5 Prozent steigern und wurde zur drittstärksten Partei. Die bisher führende Regierungspartei KESK verlor fast ein Drittel ihrer Sitze; stärkste Partei wurde mit knappem Vorsprung die KOK.

\section{Spanien: die Parlamentswahl am 20. November 2011}

Spaniens ökonomische Probleme begannen schon Ende 2007, als die Immobilienblase platzte und die Arbeitslosigkeit zu steigen begann. ${ }^{26}$ Der Minderheitsregierung der spanischen Sozialisten (PSOE) unter dem Regierungschef José Luis Rodriguez Zapatero gelang es, die Krise aus dem vom Kampf gegen den Terrorismus dominierten Wahlkampf zur Parlamentswahl im Frühjahr 2008 herauszuhalten und einen erneuten Wahlsieg zu erlangen. Danach geriet das Land jedoch schnell in eine Rezession mit explodierender Arbeitslosigkeit und rasch steigendem Haushaltsdefizit; die rivalisierende Großpartei, die konservative Partido Popular (PP), gewann die Europawahl im Juni 2009. Die in der Folgezeit verabschiedeten Maßnahmenpakete der Regierung zur Krisenbewältigung von der Erhöhung der Mehrwertsteuer im September 2009 über den Austeritätsplan Anfang 2010 und das auch auf Drängen der anderen Euro-Staaten verabschiedete drastische Sparpaket im Mai 2010 bis zu der mit einem Generalstreik der Gewerkschaften beantworteten arbeitsrechtlichen Flexibilisierung des Arbeitsmarktes und der deutlichen Senkung der Staatsausgaben im Haushaltsbeschluss vom Dezember 2010 - ließen die Umfragewerte der PSOE immer stärker

24 Vgl. Deloy, C:: General Elections in Finland, a round up one week before the election, FRS-Reihe European elections monitor, Brüssel, 2011.

25 Dies äußert sich vor allem in einem ,ausgeprägten Ethno-Nationalismus“, allerdings gepaart mit einer „eher linksorientiertern Politik in sozio-ökonomischen Fragen“, vor allem der Propagierung des nordischen Wohlfahrtsstaatsmodells (Breimaier, S.: Eine weitere rechtspopulistische Kraft in Europa, FESReihe Perspektive Nordische Länder, Berlin, 2011, 1 f.).

26 Zur Vorgeschichte der Parlamentswahl von 2011 und der Wahl selbst vgl. z.B. Martín, I./UrquizuSancho, I.: The 2011 General Election in Spain: The Collapse of the Socialist Party, in: South European Society and Politics 17/2 (2012), 347-363 und Kennedy, P.: From Unpopular Socialists to the Popular Party: The Spanish General Election of 2011, in: West European Politics 35/3 (2012), 673-681. 
sinken. Nachdem seine Partei im Mai 2011 bei den Regional- und Kommunalwahlen eine deutliche Niederlage erlitten hatte und die jüngere Generation ihrem Zorn über die verbaute Zukunft in Form einer Protestbewegung (indignados) Luft machte, verkündete Zapatero vorgezogene Neuwahlen für den November 2011.

Der von den ökonomischen Problemen dominierte Wahlkampf war ein Musterbeispiel für die Integration der Eurokrise in die bestehende parteipolitische Konfliktstruktur in Spanien: den Sozialstaatskonflikt. Die PSOE unter ihrem neuen Vorsitzenden, dem vormaligen Vize-Regierungschef Rubalcaba, präsentierte sich ,als Garant des Sozialstaates und eines funktionsfähigen öffentlichen Sektors“, im Gegensatz zur PP, die ,nur darauf warte, dem Sozialstaat den Garaus zu machen“. ${ }^{27}$ Diese Strategie konnte jedoch nicht aufgehen, da zum einen viele ihrer Anhänger der Partei vorwarfen, sie habe durch ihre sozial unausgewogenen Sparmaßnahmen ihre Grundwerte verraten, und zum anderen die überwiegende Mehrheit der Wähler der PSOE nicht zutrauten, die ökonomischen Probleme in den Griff zu bekommen. Die Schwierigkeiten der PSOE wurden noch dadurch verschärft, dass die PP eine Strategie der asymmetrischen Demobilisierung verfolgte, indem sie konsequent vermied, ihre durchaus marktfreiheitlichen und konservativen Positionen zu konkretisieren, um das PSOE-Wählerpotenzial nicht zu mobilisieren. Zudem konnte sich die u.a. die Kommunisten und Grünen umfassende Vereinigte Linke (IU-LV) mit einem auf soziale Gerechtigkeit fokussierten Wahlkampf auf Kosten der PSOE profilieren.

Die Wahl bescherte der PSOE dann auch eine drastische Niederlage: Sie erzielte ihr schlechtestes Wahlergebnis aller Zeiten und verlor ein Drittel ihrer Parlamentsmandate, während die PP zum zweiten Mal in ihrer Geschichte die absolute Parlamentsmehrheit erringen und die neue Regierung bilden konnte, die kleinen Parteien ihren Sitzanteil sogar verdoppelten.

\section{Slowakei: Die Parlamentswahl am 10. März 2012}

Im Oktober 2011 zerbrach die Regierungskoalition aus der Demokratischen und Christlichen Union (SDKÚ-DS), der wirtschaftsliberalen Freiheit und Solidarität (SaS), der Christlich-Demokratischen Bewegung (KDH) und der ungarischen Minderheitspartei ,Brücke' (MOST-HiD) an der Frage des slowakischen Beitrags zur Ausweitung der Europäischen Finanzstabilisierungsfazilität (ESFS),

27 Witte, L.: Aussichtslose Sozialisten und siegessichere Konservative, FES-Reihe Internationale Politikanalyse, Berlin, 2011, 5. 
vorgezogene Neuwahlen wurden notwendig. Premierministerin Iveta Radičová (SDKÙ-DS) hatte die ESFS-Abstimmung mit der Vertrauensfrage verbunden, um die ,kompromisslos gegen die Politik der Euro-Rettungsschirme agitierende neoliberale Koalitionspartei SaS ... zur Koalitionsdisziplin zu zwingen“. ${ }^{28}$ Diese verweigerte aber ihre Zustimmung und Radičová verlor die Abstimmung, da die größte Oppositionspartei, die europafreundlichen Sozialdemokraten (SMERSD), zwar die ESFS-Erweiterung, nicht aber die Regierung stützen wollten. ${ }^{29}$

Die SaS, für deren Vorsitzenden Richard Sulik der ESFS ,the greatest threat to Europe $^{\text {630 }}$ darstellte, wollte den Rettungsschirm, u.a. mit dem Vorschlag eines Referendums, zu einem der Hauptthemen des Wahlkampfes machen. Dies gelang jedoch nicht: Das Euro-Thema spielte ,im Wahlkampf keine relevante Rolle mehr". ${ }^{31}$ Es wurde - wie auch das innenpolitische Thema Arbeitslosigkeit durch einen gravierenden Korruptionsskandal, die sogenannte ,Gorilla-Affäre‘, verdrängt. Ende Dezember 2011 wurden mutmaßliche Geheimdienstprotokolle aus Abhöraktionen unter dem Codenamen ,Gorilla‘ veröffentlicht, die nahelegten, dass maßgebliche Parteien und Politiker in Zusammenarbeit mit der Investmentgruppe Penta die wesentlichen Privatisierungsmaßnahmen und öffentlichen Auftragsvergaben so gesteuert hatten, dass für sie maximale Vorteile heraussprangen. Die Vorwürfe, die vor allem Politiker der SDKÚ-DS und anderer Mitte-Rechts-Parteien betrafen, führten zu Massenprotesten, erschütterten das Parteienlager und führten dazu, dass eine Reihe von neu gegründeten Kleinparteien, die den Protest gegen die herrschenden politischen Zustände und vor allem den Korruptionsfilz auf ihre Fahnen geschrieben hatten, auf Wahlerfolge hofften. Dies gelang aber nur der bunt zusammengewürfelten Partei , Gewöhnliche Leute und unabhängige Persönlichkeiten' (OLaNO) die aus dem Stand zur drittstärksten Kraft wurde.

Der überragende Gewinner der Wahl war die SMER-SD, die als erste Partei seit der Unabhängigkeit des Landes bei einer Parlamentswahl die absolute Sitzmehrheit gewinnen konnte. Sowohl die $\mathrm{SaS}$ als auch die rechtspopulistischen und

28 Gehring, H./Thanei, C.: Parlamentswahlen in der Slowakei, KAS-Reihe Länderbericht, Berlin, $2012,2$. Zwei Jahre vorher, im Wahlkampf zur Parlamentswahl 2010, war die griechische Schuldenkrise eines der Hauptthemen und hatte sich die SaS strikt gegen die Rettungsmechanismen ausgesprochen.

29 Erst nach der Rücktrittsankündigung der Premierministerin ermöglichte es die SMER-SD, dass die Slowakei als letzter Staat der Eurozone die ESFS-Erweiterung mittrug.

30 Deloy, $C$.: The leftwing opposition running favourite in the early general elections in Slovakia, FRSReihe European Elections monitor, Brüssel, 2012, 3.

31 Gehring, H./Thanei, C., a.a.O., 1. Vgl. auch Petras, M./Hempel, M.: Parlamentswahlen in der Slowakischen Republik, FES-Reihe Perspektive, Berlin, 2012. 
nationalistischen Kräfte in Gestalt der Slowakischen Nationalpartei (SNS) mussten dagegen Stimmeneinbußen hinnehmen.

\section{Griechenland: die Parlamentswahlen am 7. Mai und 17. Juni 2012}

In Griechenland wurde die sich über Jahre hinweg entwickelnde, von den Regierungen verschleierte Staatsschuldenkrise nach dem durch das Versprechen steigender Sozialleistungen bewirkten Wahlsieg der Sozialisten (PASOK) im Oktober 2009 offensichtlich, als die neue Regierung ein dramatisches Haushaltsdefizit von über 12 Prozent des Bruttoinlandsprodukts verkündete. Im April 2010 bat man zur Abwendung eines Staatsbankrotts bei der Europäischen Union und dem Internationalen Währungsfonds um Hilfe, im Mai 2010 unterzeichnete die Regierung die erste, als ,Memorandum“ bekannte Kreditvereinbarung und im Oktober 2011 beschloss der EU-Gipfel ein zweites Hilfspaket, über das der innenpolitisch immer mehr in Bedrängnis geratene Premierminister George Papandreou per Referendum abstimmen lassen wollte. Der Plan scheiterte, Papandreou musste zurücktreten und einer, (Übergangs-)Regierung der nationalen Einheit' aus PASOK, der konservativen ND und der rechtspopulistischen LAOS Platz machen, wobei letztere kurz vor der Verabschiedung des zweiten Memorandums die Regierung verließ.

In dem von heftigen Bürgerprotesten gegen die von der Troika zur Bedingung für die beiden Hilfspakete gemachten Sparmaßnahmen und strukturellen Reformen begleiteten Wahlkampf zur vorgezogenen Parlamentswahl am 6. Mai 2012 ließen sich vier Gruppen im Rahmen des durch eine Reihe von Parteiabspaltungen stark zersplitterten griechischen Parteiensystem ${ }^{32}$ : (1) Das Pro-Memorandum-Lager aus den beiden - bisherigen - Großparteien PASOK und ND, die das Rettungspaket - mit der Möglichkeit kleinerer Korrekturen - als einzig realistische Krisenbewältigungsoption verteidigten, aufgrund der Schuldzuweisung für die Krise, der Ablehnung der Sparmaßnahmen und der Verdrossenheit mit der politischen Elite jedoch von massiven Stimmenverlusten bedroht waren; (2) das gemäßigte Memorandum-Lager aus der Demokratischen Linken (DIMAR), die sich für den Verbleib Griechenlands in der Eurozone und eine weniger harte Krisenbewältigungsstrategie aussprach, und der Demokratischen Allianz (DISY), die viele der im Rettungspaket vorgesehenen Reformen befürwortete, sich aber für eine stärkere Gewichtung des Wirtschaftswachstums aussprach; (3) das linke

32 Vgl. Malkoutzis, N.: Griechenlands schmerzhafter politischer Wandel, FES-Reihe Internationale Politikanalyse, Berlin, 2012. 
Anti-Memorandum-Lager aus den dogmatischen Kommunisten (KKE), die zur Abschaffung des Euro und zum Austritt Griechenlands aus der EU aufriefen, und den ehemaligen Eurokommunisten der Koalition der Radikalen Linken (SYRIZA), die nicht für einen EU-Austritt plädierte, aber der EU sehr kritisch gegenüberstand, das Memorandum als Umverteilungsinstrument von unten nach oben rundweg ablehnte und ein Schuldenmoratorium forderte; (4) schließlich das rechte Anti-Memorandum-Lager aus LAOS, die das Memorandum als Einflussinstrument ausländischer Mächte charakterisierte, der neofaschistischen Partei ,Goldene Morgenröte‘ (XA), die ebenfalls ausbeuterische ausländische Mächte und ihre griechischen Kollaborateure am Werk sah und mit aggressiven Forderungen nach Ausweisung von Immigranten aus Griechenland auftrat, und den Unabhängigen Griechen (ANEL), die das Memorandum als Verletzung der griechischen Souveränität ansahen und von Deutschland Reparationszahlungen für Schäden aus dem Zweiten Weltkrieg forderten.

Die meisten Parteien akzentuierten somit im framing der Eurokrise primär die sozio-ökonomischen Aspekte. Vom rechten Rand des Parteiensystems kamen aber auch sozio-kulturelle Argumentationslinien, die vor allem anti-deutsche Ressentiments beförderten, die auch in vielen Demonstrationen - z.B. durch die Diskreditierung Deutschlands als ,Viertes Reich“ - sichtbar wurden. ${ }^{33}$ Durch die Anti-EU-Haltung der parlamentarisch repräsentierten KKE wurde die Auseinandersetzung um die Bewältigung der Krise jedoch zum Konflikt zwischen nationaler Autonomie und supranationaler Integration, d.h. zur relevanten, eigenständigen parteipolitischen Konfliktlinie.

Die Wahl brachte die allseits erwartete Abstrafung der beiden bisher das griechische Parteiensystem dominierenden Parteien, insbesondere der PASOK, die von 43,9 auf 13,2 Prozent abstürzte, aber auch der ND, die von 33,5 auf 18,9 Prozent sank. Damit wurde sie allerdings vor dem eigentlichen Gewinner der Wahl, der von 4,6 auf 16,8 Prozent gestiegenen SYRIZA, noch zur stärksten Partei und erhielt den vom griechischen Wahlrecht vorgesehenen Bonus von 50 Parlamentssitzen. Daneben erreichten die DIMAR, KKE, ANEL und XA den Einzug ins Parlament. Nachdem mehrere Versuche zur Regierungsbildung letztlich an der Verweigerung von SYRIZA gescheitert waren, musste für den 17. Juni 2012

33 Mit Blick auf das gute Abschneiden der neofaschistischen CA bei der Wahl wurde daher in Analysen auch auf „,contradictions between Greece's rejection of the EU and Germany as the ,fourth reich 'and the support for a party with a neo-Nazi ideology" hingewiesen (Halikiopoulou, D. /Vasilopoulou, S.: Greece's response to austerity has been to say 'no to the Fourth Reich' but yes to the neo-Nazism of the Golden Dawn, in: Blogs of the LSE vom 15. Oktober 2012: http://bit.ly/SW8.JAg (18.12.2012). 
eine erneute Wahl angesetzt werden. Sie führte zu einer stärkeren Konzentration des Parteiensystems durch den Zusammenschluss von Kleinparteien bzw. deren Auflösung zugunsten der größeren. Der extrem polarisierte Wahlkampf zwischen dem von der ND geführten Lager, das den Rettungsplan vom Oktober 2011 prinzipiell unterstützte (auch wenn man ihn neu verhandeln wollte) und die Deregulierung der Wirtschaft sowie strukturelle Reformen in Aussicht stellte, und dem von SYRIZA geführten Lager, das das ,in die Hölle“ führende Memorandum annullieren, aber dennoch in der Eurozone verbleiben und Europa „auf der Basis von sozialer Kohäsion und Solidarität wiederbegründen“ wollte. ${ }^{34}$ Die ND konnte die Wahl knapp für sich entscheiden, erreichte wegen der Bonusmandate 129 Sitze (SYRIZA 71) und bildete mit der PASOK und der DIMAR die neue Regierung unter Ministerpräsident Andonis Samaras (ND).

\section{Niederlande: die Parlamentswahl am 12. September 2012}

Diese Wahl war eine vorgezogene Neuwahl, da die Minderheitsregierung aus der konservativ-liberalen VVD und den Christdemokraten (CDA) unter Duldung der rechtspopulistischen Freiheitspartei (PVV) im April 2012 an der Frage eines von der PVV abgelehnten Sparpakets gescheitert war, das gemäß der europäischen Vorgaben das durch die Finanzkrise deutlich über die 3-Prozent-Grenze gestiegene Haushaltsdefizit verringern sollte.

Im Mittelpunkt des Wahlkampfs stand der Sozialstaatskonflikt mit der Fokussierung auf wirtschafts- und sozialpolitische Themen sowie die in diesen Konflikt integrierte Debatte über die EU. Die VVD des Ministerpräsidenten Mark Rutte befürwortete eine schnelle Reduktion des Budgetdefizits durch weitere Kürzungen vor allem im Sozialbereich und Gesundheitswesen und kämpfte für einen starken Euro. Die bei der letzten Wahl zweitgrößte Partei, die sozialdemokratische PvdA, verlangte mehr Zeit für die Haushaltssanierung und legte Wert auf deren sozial gerechte Ausgestaltung. Zudem war sie für eine Neuverhandlung des im März 2012 von den EU-Staaten (mit Ausnahme von Großbritannien und Tschechien) unterzeichneten Fiskalpakts mit dem Ziel der Flankierung durch wachstumsfördernde Maßnahmen. Die Christdemokraten warben für eine größere Haushaltsdisziplin und eine europäische Bankenaufsicht. Die Partei mit der stärksten pro-europäischen Position, die linksliberale D66, forderte eine Vertiefung der europäischen Integration in Richtung politische Union.

34 So der SYRIZA-Vorsitzende Alex Tsipras, zit. n. Deloy, C.: Europe has its eyes set on the Greek general elections on 17th June, FRS-Reihe European Elections monitor, Brüssel, 2012, 2. 
Die moderaten, pro-europäischen Parteien sahen sich zwei populistischen, europakritischen bzw. -feindlichen Gruppierungen gegenüber: Die Sozialisten (SP), eine Partei mit Wurzeln in der maoistischen Bewegung der Siebzigerjahre, stellte sozialstaatliche Maßnahmen in den Mittelpunkt und wandte sich sowohl gegen strikte Haushaltsregeln und Sparmaßnahmen als auch gegen eine Vertiefung der europäischen Integration, wollte aber die EU nicht verlassen. Die PVV unter ihrem Vorsitzenden Geert Wilders, die ihre Wähler bisher mit ihren Positionen im sozio-kulturellen Libertarismus-Autoritarismus-Konflikt - vor allem ihrem Kampf gegen die „Islamisierung“ der Niederlande - mobilisiert hatte, machte diesmal die Opposition gegen Euro und EU zum Hauptthema und forderte den Austritt aus der EU und die Wiedereinführung des Gulden. ${ }^{35}$

Die Umfragen zeigten lange Zeit ein Kopf-an-Kopf-Rennen zwischen der VVD und der SP. Schwache TV-Auftritte des SP-Vorsitzenden Emile Roemer, ein furioser Schlussspurt der PvdA und die Tatsache, dass die Debatte über die EU die EU-Skepsis der Niederländer verringerte ${ }^{36}$, führten letztendlich aber dazu, dass die moderaten, pro-europäischen Parteien die Wahl gewannen - die VVD mit dem besten Ergebnis ihrer Geschichte. Die SP konnte den zwischenzeitlichen demoskopischen Höhenflug nicht in Wählerstimmen umsetzen, und für die PVV zahlte sich die Positionierung als Anti-EU-Partei mit der Forderung nach einem EU-Austritt der Niederlande nicht aus. Sie verloren ein Drittel ihrer Stimmen aus der letzten Wahl, blieben aber mit 15 Sitzen im Parlament vertreten. ${ }^{37}$ Durch die Positionierung der parlamentarisch repräsentierten PVV als Verfechterin eines EU-Austritts wurde der europäische Integrationskonflikt als neue, eigenständige Konfliktlinie zwischen nationaler Autonomie und supranationaler Integration in der parteipolitischen Konfliktstruktur der Niederlande etabliert.

\section{Italien: die Parlamentswahlen am 24./25. Februar 2013}

Bei den letzten Wahlen zu den beiden völlig gleichberechtigten, aber nach unterschiedlichem Wahlrecht bestimmten Kammern des italienischen Parlaments (Abgeordnetenhaus und Senat) im April 2008 gewann das Parteienbündnis Volk der Freiheit (PdL) mit Silvio Berlusconis Forza Italia an der Spitze die Mehrheit

35 Vgl. Deloy, C.: The Left of the Left, Running Favourite in the Dutch General Elections, FRS-Reihe European Elections monitor, Brüssel, 2012, 4.

36 Meerhof, R./van Praag, P.: EU-aanvalsplan Wilders lijkt mislukt; kiezer juist minder sceptischin, in: De Volkskrant online, 10.09.2012 (http://dpaq.de/tLkBi), 11.09.2012.

37 Der dritte Verlierer waren die Christdemokraten (2003 noch stärkste Partei, jetzt auf Platz fünf). 
in beiden Kammern. ${ }^{38}$ In der Folgezeit geriet Berlusconi wegen der horrenden Staatsverschuldung innenpolitisch sowie auf den Finanzmärkten und auf der EUEbene immer mehr unter Druck und trat schließlich im November 2011 nach einer verlorenen Parlamentsabstimmung als Ministerpräsident zurück. Es folgte eine Expertenregierung unter dem parteilosen früheren EU-Kommissar Mario Monti, die versuchte, durch einen der Bevölkerung viel abverlangenden Austeritätskurs mit harten Einschnitten bei den Staatsausgaben und Steuererhöhungen die finanzielle Konsolidierung des Landes einzuleiten und die Märkte zu beruhigen. Anfang Dezember 2012 verlor die Monti-Regierung wegen der Aufkündigung der parlamentarischen Unterstützung durch die PdL ihre Mehrheit. Daraufhin wurden die ohnehin im Frühjahr 2013 anstehenden Neuwahlen auf den 24./25. Februar vorverlegt.

Konnte man die politische Landschaft bei den letzten beiden Wahlen noch grob in ein Berlusconi-Lager und ein Anti-Berlusconi-Lager einteilen, so standen sich im Wahlkampf 2013 vier Gruppierungen gegenüber:

- die ,Bürgerliche Wahl“ Mario Montis im Bündnis mit der christlichdemokratischen UdC und zwei liberal-konservativen Kleinparteien;

- das Mitte-Links-Bündnis ,Italien. Gemeinschaftliches Eigentum“ aus Pier Luigi Bersanis Demokratischer Partei (PD), der Partei ,Linke, Ökologie, Freiheit' (SEL) und weiteren Kleinparteien;

- die ,Mitte-Rechts-Koalition“ aus Berlusconis PdL, der rechtspopulistischen Lega Nord und weiteren, teils rechtsextremistischen Kleinparteien; sowie

- $\quad$ die 2009 gegründete Protestliste ,Bewegung 5 Sterne“ des TV-Komikers und Bloggers Beppe Grillo.

Letztlich ging es in dem von der Staatsschuldenkrise und den negativen sozialen Folgen der Sparpolitik dominierten Wahlkampf jedoch um die Alternative ,,proeuropäischer Stabilitätskurs oder europaskeptische Rebellion“. 39 Das von Monti geführte Bündnis setzte auf einen klar pro-europäischen Kurs, wollte den von der Regierung begonnenen Sparkurs fortführen, den Haushalt weiter sanieren und die Strukturreformen vor allem durch weitere Liberalisierungsschritte auf dem Arbeitsmarkt vertiefen. Auch das europafreundliche Mitte-Links-Bündnis unter Pier Luigi Bersani plante den Sparkurs der Monti-Regierung grundsätzlich bei-

38 Die Wahlen erfolgen nach der Proporzregel mit einem Mehrheitsbonus für die stärkste Gruppierung, der im Abgeordnetenhaus gemäß des nationalen Resultats, im Senat jedoch Region für Region vergeben wird, so dass unterschiedliche Mehrheiten in beiden Häusern möglich sind.

39 Braun, M.: Italien vor den Wahlen, FES-Reihe Perspektive, Berlin, 2013, 3. 
zubehalten, ihn aber durch neues Austarieren der Krisenlasten sozialverträglicher zu gestalten und neben den Sparmaßnahmen auch Wachstumsimpulse zu setzen. Statt weiterer Privatisierung sollten gemeinschaftliches Eigentum geschaffen, der Markt besser reguliert, die Mittelschicht entlastet, die Reichen stärker zur Kasse gebeten und Bildung und Forschung durch Investitionsprogramme gefördert werden. Man sah das Vertrauen der EU und der Finanzmärkte als wesentliche Voraussetzung für den Konsolidierungsprozess an und setzte sich für einen starken Euro ein.

Silvio Berlusconi, dem sowohl sein Gespür für die Ängste und Sehnsüchte des kleinen Mannes als auch seine Medienmacht und die professionelle WahlkampfMaschinerie zugute kamen, setzte dagegen mit aggressiv-populistischer Rhetorik auf einen europakritischen und anti-deutschen Wahlkampf und mobilisierte die Wähler innenpolitisch mit einem „Feuerwerk populistischer Versprechungen“ ${ }^{40}$ Er machte den falschen Kurs der EU für die italienische Krise verantwortlich, drohte, unter Umständen die Eurozone zu verlassen, warf der Monti-Regierung vor, sie habe die „Germanisierung der italienischen Politik und ihre Transformation in ein deutsches Protektorat ${ }^{\star 41}$ akzeptiert, verurteilte ihre rigide Austeritätspolitik, wollte einen Großteil ihrer Maßnahmen rückgängig machen und versprach eine Reihe von Steuererleichterungen. Insbesondere wollte er die von Monti eingeführte Steuer für Immobilienbesitzer abschaffen und den Leuten das gezahlte Geld in bar zurückzahlen, ein sehr zugkräftiges Versprechen angesichts der Tatsache, dass 80 Prozent der Italiener im eigenen Heim wohnen. Auch das ,Movimento 5 Stelle', eine „basisdemokratische Bewegung mit charismatischer, ja diktatorischer Führung ${ }^{642}$ durch Beppe Grillo, die als Antiparteien-Partei mit zahlreichen Vorschlägen zur Abschaffung von Privilegien der politischen Klasse vor allen jüngere Protestwähler, Politikverdrossene und Desillusionierte ansprach, positionierte sich dezidiert europakritisch und favorisierte ein Referendum über den Verbleib Italiens in der Eurozone.

Das Ergebnis der Parlamentswahlen führte zu einem „unregierbaren Italien“ ${ }^{43}$ Bersanis Mitte-Links-Allianz erhielt bei der Abgeordnetenhauswahl zwar weniger als 30 Prozent der Stimmen und lag damit nur knapp vor der von Berlusconi

40 Plate, K.C.: Italien im Wahlkampf, KAS-Reihe Länderbericht, Berlin 2013. 2.

41 Zit. n. Deloy, C.: Parliamentary Elections in Italy: an election with several unknown elements, FRSReihe European elections monitor, Brüssel 2013, 4.

42 Braun, M.: Wenn ein Komiker den Nerv trifft, in: taz online, 13.02.2013.

43 Deloy, D.: The Parliamentary Elections lead to an ungovernable Italy, FRS-Reihe European elections monitor, Brüssel 2013,1. 
geführten Koalition, erreichte aber aufgrund des Mehrheitsbonus die Mandatsmehrheit. ${ }^{44}$ Im gleichberechtigten Senat jedoch konnte sie nur 123 der 315 Sitze erobern und hat nicht einmal zusammen mit dem grundsätzlich koalitionsbereiten Bündnis Montis, das nur 19 Sitze bekam, eine Mehrheit. Da keines der beiden Parteienbündnisse eine Koalition mit Silvio Berlusconi eingehen wollte und die ,Bewegung 5 Sterne' Koalitionen mit den etablierten Parteien grundsätzlich ablehnte, scheiterte der zunächst mit der Regierungsbildung beauftragte Pier Luigi Bersani. Danach setzte Staatspräsident Giorgio Napolitano eine zehnköpfige Expertenkommission aus Vertretern der Politik, Wirtschaft und Justiz ein, die auf Grundlage der Parteiprogramme institutionelle und ökonomische Reformen erarbeiten und damit eine Annäherung der großen Parteien unterstützen sollte. Auch diese Kommission brachte jedoch keinen Durchbruch. Nachdem auch die turnusmäßige Neuwahl des Staatspräsidenten mehrfach misslungen war, stellte sich Napolitano, der eigentlich abtreten wollte, doch noch einmal zur Verfügung und beauftragte nach seiner Wiederwahl den stellvertretenden PDVorsitzenden Enrico Letta mit der Regierungsbildung. Dieser schaffte es schließlich, eine breite Koalition aus der PD, Montis Zentrumspartei und Berlusconis PdL zu bilden, die jedoch - vor allem aufgrund wiederholter Drohungen Berlusconis, die Unterstützung zu entziehen - auf schwachen Beinen steht.

\section{Fazit}

Die Eurokrise führte in sechs der sieben untersuchten Länder zu einer Politisierung des Themas europäische Integration und beeinflusste den nationalen Parteienwettbewerb deutlich (vgl. unter Tab. 1). Lediglich in der Slowakei, wo die Regierung an der Frage des slowakischen Beitrags zur Ausweitung der EuroRettungsschirme zerbrach, spielte das Euro-Thema im Wahlkampf keine relevante Rolle mehr, da es durch einen gravierenden Korruptionsskandal verdrängt wurde.

Stellt man auf die Frage ab, ob durch die Eurokrise und ihre Folgen eine neue, eigenständige Konfliktlinie in der nationalen parteipolitischen Konfliktstruktur etabliert wurde, muss man der These vom ,limited impact of Europe on national party systems ${ }^{645}$ heute differenziert betrachten. In zwei der sieben untersuchten

44 Die von Berlusconi geführte Koalition bekam 20 Prozent, die „Bewegung 5 Sterne“ 17 Prozent und Montis Bündnis 8 Prozent der Abgeordnetenhausmandate.

45 Mair, P.: The Limited Impact of Europe on National Party Systems, in: West European Politics 23/4 (2000), 27-51. 
Länder der Eurozone ${ }^{46}$ (in Griechenland und den Niederlanden) hat die Eurokrise zur Etablierung des europäischen Integrationskonflikts als eigenständige Konfliktlinie zwischen nationaler Autonomie und supranationaler Integration in der parteipolitischen Konfliktstruktur geführt, indem sich zwei parlamentarisch repräsentierte Parteien (die griechische KKE und die niederländische PVV) im Wahlkampf als Anti-EU-Partei positionierten, die den Austritt ihres Landes aus der EU zu ihrer zentralen Wahlkampfbotschaft erhoben. ${ }^{47}$

Tabelle 1: Das framing der Eurokrise in den untersuchten Wahlkämpfen

\begin{tabular}{l|c|c|c}
\hline Land & $\begin{array}{c}\text { Integration in den } \\
\text { sozio-ökonomi- } \\
\text { schen Sozialstaats- } \\
\text { konflikt? }\end{array}$ & $\begin{array}{c}\text { Integration in den } \\
\text { sozio-kulturellen } \\
\text { Libertarismus- } \\
\text { Autoritarismus- } \\
\text { Konflikt? }\end{array}$ & $\begin{array}{c}\text { Etablierung einer } \\
\text { eigenständigen } \\
\text { Konfliktlinie? }\end{array}$ \\
\hline Irland & ++ & & \\
\hline Finnland & + & & - \\
\hline Spanien & ++ & - & ++ \\
\hline Slowakei & - & & ++ \\
\hline Griechenland & & & \\
\hline Niederlande & ++ & + & + \\
\hline Italien & & & \\
\hline
\end{tabular}

Anmerkungen: $++/+=$ starke/weniger starke Thematisierung im Wahlkampf; - = Eurokrise spielte im Wahlkampf keine relevante Rolle.

In den vier anderen Ländern wurde der Konflikt zwischen den Parteien um ihre unterschiedliche Position zur Eurokrise und dessen nationale Bewältigung in die bestehende nationale Konfliktstruktur integriert. Das framing des Themas durch die Wahlkampfkommunikation der Parteien erfolgte, der Natur des Problems gemäß, vor allem durch die Akzentuierung der sozio-ökonomischen Aspekte mit der Folge einer Integration in den sozio-ökonomischen Sozialstaatskonflikt.

46 Interessant wäre auch eine Ausweitung der Analyse auf die nicht der Eurozone angehörenden EUMitgliedstaaten durch die Fragestellung, inwieweit die Krise dort zu einer Veränderung des framings der EU-Debatte in der nationalen parteipolitischen Konfliktstruktur geführt hat. Zu denken wäre etwa an Großbritannien und den Einfluss der aufgrund des britischen Wahlrechts zwar nicht im Parlament vertretenen, aber in der öffentlichen Diskussion durchaus präsenten Anti-EU-Partei UKIP auf die europapolitische Positionierung der regierenden Conservative Party.

47 Mit dem Verweis auf die Haltung der Sinn Féin könnte man auch noch Irland zu dieser Gruppe zählen. Da die SF mit Blick auf ihre Geschichte aber keine Anti-EU-Partei ist und die EU-Austrittsforderung in ihrem Wahlkampf eine nur marginale Rolle spielte, wird hier davon abgesehen. 
Einige Parteien haben mit dem Schüren der Angst vor nationalem Souveränitätsverlust und ausländischen Eingriffen durchaus aber auch sozio-kulturelle Aspekte der Eurokrise thematisiert. Zudem machte die Analyse die schon des Öfteren empirisch belegte Tatsache deutlich, dass Parteien am linken wie am rechten Rand des politischen Spektrums - aus unterschiedlichen Gründen - europakritisch bzw. europafeindlich eingestellt sind. ${ }^{48}$ 